\title{
Topology optimization of nanoparticles for localized electromagnetic field enhancement
}

Christiansen, Rasmus Ellebæk; Vester-Petersen, Joakim; Madsen, Søren Peder; Sigmund, Ole

Published in:

Proceedings of the 2017 International Conference on Numerical Simulation of Optoelectronic Devices (NUSOD)

Link to article, DOI:

10.1109/NUSOD.2017.8009962

Publication date:

2017

Document Version

Peer reviewed version

Link back to DTU Orbit

Citation (APA):

Christiansen, R. E., Vester-Petersen, J., Madsen, S. P., \& Sigmund, O. (2017). Topology optimization of nanoparticles for localized electromagnetic field enhancement. In Proceedings of the 2017 International Conference on Numerical Simulation of Optoelectronic Devices (NUSOD) (pp. 3-4). IEEE. https://doi.org/10.1109/NUSOD.2017.8009962

\section{General rights}

Copyright and moral rights for the publications made accessible in the public portal are retained by the authors and/or other copyright owners and it is a condition of accessing publications that users recognise and abide by the legal requirements associated with these rights.

- Users may download and print one copy of any publication from the public portal for the purpose of private study or research.

- You may not further distribute the material or use it for any profit-making activity or commercial gain

- You may freely distribute the URL identifying the publication in the public portal 


\section{Topology optimization of nanoparticles for localized electromagnetic field enhancement}

\author{
Rasmus E. Christiansen (a) \\ Joakim-Vester Petersen and Søren Peder Madsen, (b) \\ Ole Sigmund (a)
}

(a) DTU Mekanik, Department of Mechanical Engineering

Technical University of Denmark

Kongens Lyngby, Denmark

(b) Department of Engineering - Nanoengineering

Aarhus University

Aarhus C, Denmark

Mail: raelch@mek.dtu.dk

\begin{abstract}
We consider the design of individual and periodic arrangements of metal or semiconductor nanoparticles for localized electromagnetic field enhancement utilizing a topology optimization based numerical framework as the design tool. We aim at maximizing a function of the electromagnetic field amplitude in a region of space through the introduction of nanoparticles in and/or near the region.
\end{abstract}

Keywords - Topology optimization; Electromagnetics; Nanoparticles; Field enhancement; Numerical simulations

\section{INTRODUCTION}

This work concerns the development and application of a topology optimization [1] based numerical framework for designing individual and periodic arrangements of extruded 2D and 3D nanoparticles for spatially localized field enhancement. The nanoparticles are designed to manipulate and amplify the electromagnetic field in a region of space by taking advantage of various physical mechanisms including refraction, reflection and diffraction of the field from particles, which focus the field and enable resonance phenomena as well as plasmonic effects capable of highly localized field amplification. The electromagnetic field is calculated from Maxwell's equations in the frequency domain and the goal is maximizing the field enhancement by designing the topology of nanoparticles. Hence, rather than developing a model which targets a specific physical mechanism we allow the particles to make use of multiple mechanisms at once without restrictions.

Density based topology optimization as a design tool allows for a very large number of design variables which provides high flexibility of the shape and topology of the design, essentially only limited by the resolution of the numerical model and production techniques used to fabricate the optimized designs. There is no requirement of an a priori knowledge of the nanoparticle geometry, nor are there any limits on imposing such knowledge if available. A description of topology optimization for nanophotonics is found in [2].

There exist numerous applications for which it is desirable to be able to enhance the electromagnetic field in a region of space. The amplification and detection of a weak signal and the facilitation of a physical process depending on the local field

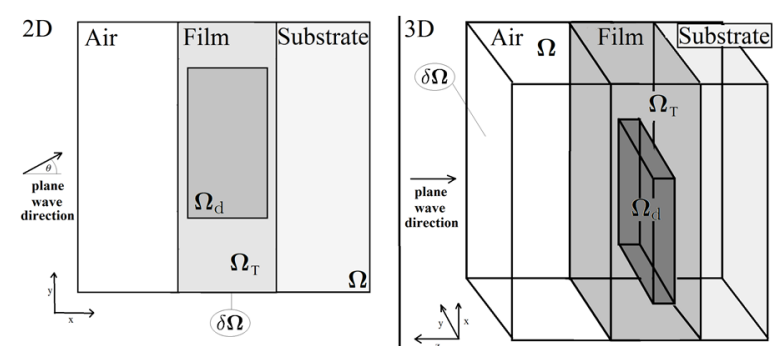

Figure 1. Sketch of model problem domain in 2D and 3D. $\Omega, \Omega_{\mathrm{d}}$ and $\Omega_{\mathrm{T}}$ denotes the model, target and design domains respectively, $\delta \Omega$ denotes the model domain boundary.

magnitude, such as the excitation of electron states, can be mentioned as examples. As an application, we consider a problem of enhancing the local intensity of infrared light in a $\mathrm{TiO}_{2} \mathrm{Er}^{3+}$ medium by introducing $\mathrm{Au}$ and $\mathrm{Si}$ nanoparticles on top of or inside the medium. This problem is relevant for the improvement of the efficiency of solar cells as a large field strength is required to enable the conversion of photons having energy below the band gap to higher energy in order to be able to generate electricity in the cell. [3]

\section{Modelling APPROACH}

The problem of designing the nanoparticles is cast as a continuous optimization problem with the goal of maximizing a prescribed objective functional, $\Phi$, depending on the electric field, E, see eq. (1), in a target region by manipulating the topology of nanoparticles near or inside the region.

$$
\Phi=\int g(\mathbf{E}) d \Omega_{\mathrm{T}}
$$

Here $g$ is some functional of $\mathbf{E}$. The physics is modelled in the frequency domain using Maxwell's equations where $\mathbf{E}$ is obtained by solving eq. (2) in a model domain consisting of a square (2D) / cubic (3D) region of space, see Figure 1, for either simple periodic or first order absorbing boundary conditions, enabling the study of periodic arrangements of nanoparticles as well as single nanoparticles.

$$
\nabla \times(\nabla \times \mathbf{E})-\epsilon_{r} k^{2} \mathbf{E}=0
$$

Here $\epsilon_{r}$ denotes the relative electric permittivity and $k$ denotes the wave number. The nanoparticles are modelled using a material distribution field, $\xi$, which in turn is used to interpolate $\epsilon_{r}$ in eq. (2), between the background and nanoparticle materials.

The optimization problem is formulated as a $\min / \max$ problem where the design variables are introduced through $\xi$ and where multiple frequencies, $f$, and polarizations, $p$, of the electromagnetic field may be considered simultaneously.

$$
\begin{aligned}
& \max _{\xi} \min _{f, p} \Phi\left(\mathbf{E}\left(f, p, \epsilon_{r}(\xi)\right), \xi\right) \\
& \text { where } \mathbf{E} \text { is a solution to eq. (1) }
\end{aligned}
$$




$$
\text { s.t. } 0 \leq \xi \leq 1
$$

A set of filtering techniques are used to assure physically admissible final designs [4,5].

\section{COMPUTATIONAL APPROACH}

To obtain an approximation of the electromagnetic field, eq. (2) is discretized using the finite element method utilizing standard first order finite elements in 2D and first order Nedelec elements in 3D [6]. The developed design framework is implemented in MATLAB in 2D and in $\mathrm{C}++$ in 3D taking advantage of the PETSc framework [7] for parallelization to enable large scale simulations. The indefinite nature of eq. (2) means that a direct solver (MUMPS) is used to solve the discretized equation system. This effectively limits the size of the equation system it is possible to consider in a feasible time frame to approximately 6 million degrees of freedom. The 3D framework allows for the embarrassingly parallel solution of multiple finite element problems simultaneously. This enables the simultaneous consideration of multiple frequencies, polarizations of the field and design realizations for the design problem without a significant increase of the optimization problem solution time.

The material distribution field, $\xi$, is discretized into a piecewise constant field with one design variable in each finite element and the optimization problem, eq. (3), is solved iteratively using the gradient based optimization algorithm, the Globally Convergent Method of Moving Asymptotes (GCMMA) [8]. To enable the use of GCMMA the sensitivities of the objective function with respect to the design variables must be calculated. This is done using the adjoint approach [9].

\section{Preliminary Result}

As an example of the application of the framework in 2D consider the design of a periodic array of extruded $\mathrm{Si}$ nanorods embedded in a $\mathrm{TiO}_{2}$ film on top of a $\mathrm{SiO}_{2}$ substrate subject to a TM polarized electromagnetic wave at normal incidence to the film at a wavelength of $1500 \mathrm{~nm}$. The goal is to maximize the integral of $g=|\mathbf{E}|^{3}$ in the $\mathrm{TiO}_{2}$ film at this wavelength and polarization. Figure 2 shows the final design along with field plots of $|\mathbf{E}|^{3}$ for plane wave excitation from the left boundary with and without the Si rods. Figure 3 shows the amplification as a function of wavelength and angle. A value of $\sim 390$ is achieved at $1500 \mathrm{~nm}$. While the amplification is seen to be only moderately sensitive to angular variations it is observed to be frequency sensitive with a FWHM of $\sim 7 \mathrm{~nm}$.

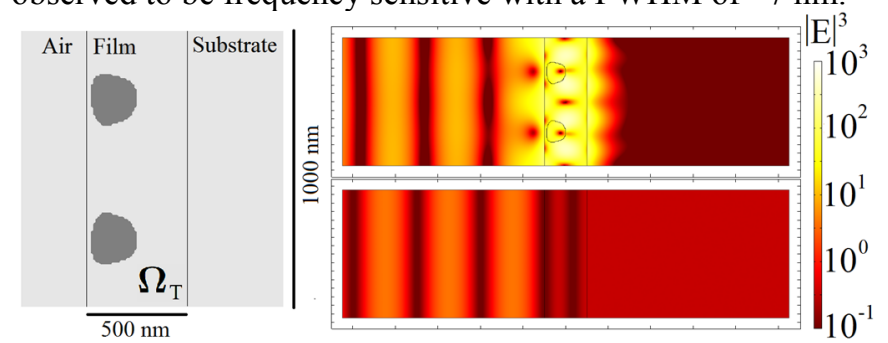

Figure 2. (Left) Si nanorod design in dark gray. (Right) $|\mathrm{E}|^{\wedge} \mathbf{3}$ field on $\log _{10}$-scale in and around the film under TM plane wave excitation at $1500 \mathrm{~nm}$ (top) with and (bottom) without the Si rods.
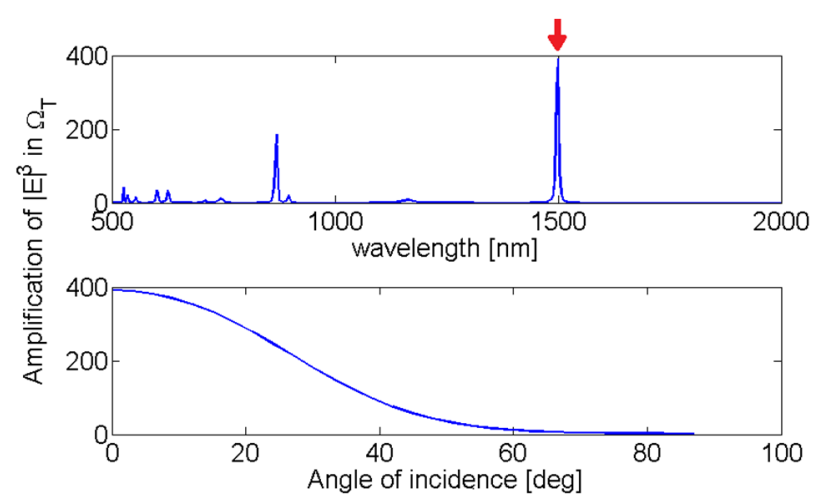

Figure 3. $|\mathbb{E}|^{\wedge} 3$ amplification in $\Omega_{\mathrm{T}}$ as a function of (top) wavelength, (bottom) plane wave angle of incidence relative to normal incidence.

\section{Discussion}

The example above was optimized for a single frequency and the amplification of $\Phi$ of $\sim 390$ is indeed significant. The sensitivity of design towards frequency variations makes it ill unsuited for broadband use. This sensitivity may however be reduced by including several frequencies in the design problem at the potential cost of a lower amplification at each individual frequency. Additional considerations, such as sensitivity to unit cell size, film thickness and perturbations of the design geometry are also relevant and straightforward to consider directly in the design problem using the developed framework. The design framework provides a platform for designing nanoparticles for a range of applications where spatially localized field enhancement is desired.

\section{ACKNOWLEDGMENTS}

The authors acknowledge the support from the Innovation Fund Denmark under the project SunTune.

\section{REFERENCES}

[1] Bendsoe, Martin Philip, and Ole Sigmund. Topology optimization: theory, methods, and applications. Springer Science \& Business Media, 2013. [2] Jensen, Jakob Søndergaard, and Ole Sigmund. "Topology optimization for nano-photonics." Laser \& Photonics Reviews 5.2 (2011): 308-321.

[3] Goldschmidt, Jan Christoph, and Stefan Fischer. "Upconversion for photovoltaics-a review of materials, devices and concepts for performance enhancement." Advanced Optical Materials 3.4 (2015): 510-535.

[4] Wang, Fengwen, Boyan Stefanov Lazarov, and Ole Sigmund. "On projection methods, convergence and robust formulations in topology optimization." Structural and Multidisciplinary Optimization 43.6 (2011): 767-784.

[5] Christiansen, Rasmus E., et al. "Creating geometrically robust designs for highly sensitive problems using topology optimization." Structural and Multidisciplinary Optimization 52.4 (2015): 737-754.

[6] Jin, Jianming. Electromagnetic analysis and design in magnetic resonance imaging. Vol. 1. CRC press, 1998.

[7] Balay, Satish, et al. "PETSc users manual revision 3.3." Computer Science Division, Argonne National Laboratory, Argonne, IL (2012).

[8] Svanberg, Krister. "A class of globally convergent optimization methods based on conservative convex separable approximations." SIAM journal on optimization 12.2 (2002): 555-573.

[9] Tortorelli, Daniel A., and Panagiotis Michaleris. "Design sensitivity analysis: overview and review." Inverse problems in Engineering 1.1 (1994): 71-105. 